\title{
Impulsiveness, Behavioral Disorders and Alcohol Misuse in Teenage Students in Northern Italy
}

Michela Gatta $^{1 *}$, Andrea Spoto ${ }^{2}$, Serena Lamboglia ${ }^{1}$, Marco Penzo ${ }^{1}$, C.Paolo Testa ${ }^{1}$ and Pier Antonio Battistella ${ }^{1}$

${ }^{1}$ Woman and Child Care Department, Padua University Hospital, Italy

${ }^{2}$ Department of General Psychology, Padua University, Italy

\begin{abstract}
Introduction: This study aimed to analyze the growing phenomenon of adolescent alcohol consumption and its association with behavioral disorders, focusing particularly on the "impulsiveness" trait and seeking any gender-related differences.
\end{abstract}

Subjects and methods: The sample consisted of 273 secondary school pupils (in $9^{\text {th }}$ to $13^{\text {th }}$ grade), 140 of them males and 133 females, with a mean age of 15.4 years \pm 1.1 SD.

The following tests were administered:

- The Barratt Impulsiveness Scale (BIS-11) to measure their impulsiveness;

- The Youth Self Report 11-18 (YSR) to identify any psychobehavioral problems;

- The Adolescents' Saturday Nights Questionnaire (QAS) (Questionario Adolescenti Sabato Sera) (Gallimberti et al, 2011) to obtain information on the modality and quantity of their alcohol consumption.

Results: While a greater degree of impulsiveness was clearly associated with alcohol consumption, heavier drinkers were not more impulsive than more moderate drinkers. This would seem to confirm the hypothesis that a tendency for impulsiveness predisposes to alcohol consumption. On the other hand, our data indicate a higher prevalence of behavioral disorders among heavy drinkers than among more moderate drinkers: the higher the score on the behavioral disorder scales, the higher the alcohol consumption. This picture could represent the behavioral correlates associated with impulsiveness, exacerbated by the neurobiological effects of alcohol on the brain and particularly on the frontal regions (still immature in preadolescence and adolescence), i.e. the site of the functions that are altered in behavioral disorders.

The 'gender' variable did not influence the relationship between alcohol consumption and impulsiveness in our sample, but the two genders seemed to differ in their susceptibility to different subdomains of impulsiveness and behavioral disorders, i.e. non-planning impulsiveness with conduct disorder in males and motor impulsiveness with oppositional-defiant disorder in females.

Conclusion: This finding is of interest because it enables us to link the use and effects of alcohol in adolescence with certain psychopathologies and to identify a possibly alcohol-related tendency of one or other gender to develop a given disorder.

Keywords: Alcohol misuse; Behavioral disorders; Adolescents; Impulsive behavior

\section{Background}

The neuronal remodeling characteristic of the brain's development in adolescence extensively involves the prefrontal cortex, which is believed to be implicated in memory, voluntary motor activity, impulse control, learning rules, spatial learning, and decision-making [1-4]. In particular, decision-making capacity relies on an exchange between the prefrontal cortex and the amygdala, and in adolescence it reflects a sort of imbalance in the rate of maturation of the cortical regions: areas performing more basic functions mature earlier, while those involved in higher-order functions mature later. The prefrontal cortex, which handles reasoning and other 'executive' functions, emerges late in our development and is among the last to mature, and this explains the propensity of adolescents to be impulsive and take risky decisions, including those relating to the use of psychoactive drugs such as alcohol $[5,6]$. The worrying increase in alcohol consumption in developmental age [7-9] coincides with reports on the harmful effects of ethanol on the brain, which are known to be more severe in young people because they interfere with the brain's ongoing maturation processes and can lead to severe cognitive and behavioral impairments. The dysfunctions caused by alcohol abuse are especially harmful on a level with the frontal and prefrontal areas, and can give rise to psychobehavioral correlates such as attention disorders and decision-making problems, failure to inhibit inappropriate behavior and difficulty in delaying gratification $[10,11]$, all important facets of the multifactorial construct of impulsiveness.

Functional and neuroimaging studies have contributed to a more in-depth understanding of the adolescent brain's development, enabling the identification of deficiencies affecting the cerebral system that are caused by alcohol use. Most studies in this field have focused on the phenomenon of binge drinking, demonstrating early and overall electrophysiological impairments that influence perception and attention, and the cognitive stage relating to decision-making [12]. In particular, there is evidence of an impaired capacity for inhibitory control, making it hard for individuals to control their impulses [13]. It is worth noting that similar alterations are detectable in subjects who

*Corresponding author: Dr. Gatta Michela, Woman and Child Care Department, Via Colli 4, 35143 Padova, Italy, Tel: 0039 (0) 49 8217690; Fax: 0039 (0) 49821 7708; E-mail: michela.gatta@unipd.it

Received May 31, 2014; Accepted June 28, 2014; Published July 08, 2014

Citation: Gatta M, Spoto A, Lamboglia S, Penzo M, Testa CP, et al. (2014) Impulsiveness, Behavioral Disorders and Alcohol Misuse in Teenage Students in Northern Italy. J Psychol Abnorm Child 3: 122. doi:10.4172/2329-9525.1000122

Copyright: ( 2014 Gatta M, et al. This is an open-access article distributed under the terms of the Creative Commons Attribution License, which permits unrestricted use, distribution, and reproduction in any medium, provided the original author and source are credited. 
have conduct disorder whose impulsiveness becomes apparent, from a neurocognitive standpoint, in tasks involving the need to inhibit motor response [14] and in decision-making tasks when individuals need to integrate changes in reinforcement and punishment contingencies in their decisions [15-18]. From the morphometric standpoint, changes affecting brain structures like the amygdala and the ventromedial prefrontal cortex are already identifiable in preadolescents and adolescents with antisocial conduct [19-21].

Based on these premises, it is clear that the relationship between impulsiveness, behavioral disorders and alcohol intake in young people is an area of research that warrants further analysis, with potential implications for primary and secondary prevention efforts, given the damage that ethanol can cause in the still-developing brain.

\section{Aim}

The present study focuses on the functional and behavioral correlates of the neurobiological changes induced by ethanol on the developing brain, with specific reference to impulsiveness and behavioral disorders. In general, our aim was to compare the literature on adults concerning the relationship between impulsiveness and alcohol consumption with data emerging from a case study on adolescents. In particular, the study investigated in a sample of adolescents whether, and to what degree alcohol consumption was associated with traits of impulsiveness and/ or aggressive/impulsive behavior, also considering any gender-related differences.

\section{Methods}

\section{Sample}

The sample consisted of 273 secondary schools pupils ( 60 attending grammar schools, 126 at technical colleges, and 87 at vocational schools), comprising 140 males and 133 females, between 13 and 19 years of age (mean 15.4 years $\pm 1.1 \mathrm{SD}$ ). The schools involved were all in the province of Padua, in the Veneto region of north-east Italy, and had volunteered to take part in the study subject to the students' and their parents' consent.

\section{Procedures}

The questionnaires were administered in the second week of October 2011. The date was chosen because it was far away from any public holidays or particular events that might prompt an unusual alcohol consumption, in order to investigate the situation on any typical Saturday evening. The questionnaires were kept anonymous by using acronyms for pairing the two tools. Informed verbal consent to the students' participation was required first from the school director, then from all parents and from each student enrolled. None of the parents or students refused. The study was conducted in full compliance with the Padua General Hospital's ethical principles (Deliberation of the Ethical Committee, dated 13.02.2012).

\section{Tools}

The sample of students was administered three questionnaires: the Barratt Impulsiveness Scale-11 (BIS-11) to measure impulsiveness, the Youth Self Report in the version for 11-18 year-olds (YSR 11-18) to identify elements of psychopathological vulnerability, and an ad hoc questionnaire on how adolescents spent their Saturday evenings (Questionnaire on Adolescents' Saturday nights - QAS) to obtain information on their modalities and quantities of alcohol consumption.

The Barratt Impulsiveness Scale-11 (BIS-11) [22] is a widelyused measure of impulsiveness. It includes 30 items that are scored to yield six first-order factors (attention, motor, self-control, cognitive complexity, perseverance, and cognitive instability impulsiveness) and three second-order factors (attentional, motor, and non-planning impulsiveness) [22]. Initially developed in the United States, the BIS-11 has since been applied all over the world, including Italy with reported internal consistency coefficients for the BIS-11 total score in the range of 0.79 to 0.83 for separate populations of undergraduates, substance abusers, general psychiatric patients, and prison inmates.

The YSR 11-18 [2] is one of the most commonly-used scales for rating juvenile behavior and it is used internationally in the clinical setting and in research. It is in the form of a questionnaire completed by adolescents, and has been translated and validated for Italians too $[2,23]$. The questionnaire yields two profiles: one for competences (activities, social functioning, school performance), the other for behavioral and emotional problems, which can be assessed as "normal", "borderline" or "clinical" on 8 specific syndrome scales. The syndrome scales relating to the various psychopathological pictures are: anxiety/ depression, withdrawal, somatization, social problems, thoughtrelated problems, attention problems, aggressive and rule-breaking behavior. The problems are grouped into: internalizing problems (anxiety, depression withdrawal somatization); externalizing problems (aggressive and rule-breaking behavior); and other problems (social problems, thought-related problems, attention problems). The DSM IV oriented scales of the YSR 11-18 were considered in this study, and specifically those referring to externalizing behavioral disorders, i.e. conduct problems, oppositional-defiant problems and attention deficit/ hyperactivity problems.

The QAS, consisting of questions with multiple-choice answers, was prepared for the purpose of exploring the consumption of recreational drinks, both alcoholic and non-alcoholic, during the previous Saturday evening (intended as the hours elapsing between dinner-time and bedtime); it had been used in other study aiming to explore juvenile alcohol consumption and related factors [7-9]. The questionnaire enabled us to collect information on the quantity of alcoholic and soft drinks ingested by our sample during the evening. Using the results obtained with the QAS, our students were divided according to their self-reported alcohol-drinking habits into three groups: non-drinkers (0 alcohol units $[\mathrm{AU}])$, moderate drinkers (1-2 AU), and heavy drinkers ( $\geq 3 \mathrm{AU}$ ). The term alcohol unit (AU) was used in the sense of the number of drinks containing approximately $12 \mathrm{~g}$ of ethanol (corresponding to a small glass of medium-bodied wine, a can of beer, or a shot of spirit).

The impulsiveness trait was interpreted on the strength of the results of the BIS-11, focusing on the three subscales concerning motor impulsiveness, non-planning impulsiveness, and attentional or cognitive impulsiveness (these subscales are combined to form the BIS TOT): the higher the score on the BIS 11, the greater the level of impulsiveness identified [24].

As concerns aggressive-impulsive behavior, this was measured from the results of the YSR 11-18, with a particular focus on the DSM IV oriented scales relating to oppositional-defiant disorder (YSRODD), conduct disorder (YSR-CD), and attention-deficit/hyperactivity disorder (YSR-ADHD): here again, the higher the score in the YSR, the greater the behavioral problems identified.

\section{Data Analyses}

The data were analyzed using the SPSS 20 statistical software. Univariate analyses of variance (ANOVA) were used to test our research hypotheses, alongside contrast analyses to check for differences between subsamples. The variable used in the grouping for ANOVA 
and the subsequent contrast analyses was the consumption of alcohol units classified as: $0 \mathrm{AU} ; 1-2 \mathrm{AU} ; \geq 3 \mathrm{AU}$.

\section{Results}

In our sample 147 persons (53.8\%) were non-drinkers, 99 (36.3\%) were moderate-drinkers, while the remaining 27 (9.9\%) individuals were heavy drinkers.

The $10.8 \%$ of our sample scored above the clinical cut-off in YSRADHD, the $18.6 \%$ in YSR-ODD and the $11.5 \%$ in YSR-CD. Means and standard deviations for the four scales of BIS-11 and for the three scales of YSR considered in this study are reported in Table 1.

A comparison between males and females with respect to the prevalence of alcohol use and to the scores obtained at the subscales of BIS-11 and YSR was conducted and results are reported in Table 2.

Results showed significant differences between genders only for YSR-ADHD and YSR-CD scales where males scored higher than females. Furthermore, it emerged that males tend to drink more than females.

We sought an association between alcohol consumption and impulsiveness and/or aggressive-impulsive behavior. Our data showed that a higher alcohol consumption coincided with a higher score in

\begin{tabular}{|c|c|c|}
\hline Variable & Mean (sd) & Cut-off \\
\hline BIS-ImpAtt & $17.13(3.38)$ & *Range: $8-40$ \\
\hline BIS-ImpMot & $21.13(3.62)$ & ${ }^{*}$ Range: $11-55$ \\
\hline BIS-ImpNPlan & $27.73(4.49)$ & ${ }^{*}$ Range: $11-55$ \\
\hline BIS-TOT & $65.87(8.46)$ & *Range: $30-120$ \\
\hline YSR-ADHD & $55.22(7.57)$ & 64 \\
\hline YSR-ODD & $50.86(6.42)$ & 64 \\
\hline YSR-CD & $56.38(8.23)$ & 64 \\
\hline
\end{tabular}

Table 1: Means (standard deviations) and cut-offs for the subscales included in the study. * No cut-offs are available for BIS-11. Thus, the potential range of scores is reported in order to allow a better understanding of the observed values.

\begin{tabular}{|c|c|c|c|c|}
\hline Variable & Mean (sd) Males & Mean (sd) females & Test statistic Value & $\mathbf{p}$ \\
\hline Alcohol Use & Mean Rank* 147.09 & Mean Rank ${ }^{*} 126.38$ & $\mathrm{Z}=-2.43$ & $<.05$ \\
\hline BIS-ImpAtt & $17.38(3.52)$ & $16.87(3.22)$ & $\mathrm{t}_{255}=1.21$ & $\mathrm{~ns}$ \\
\hline BIS-ImpMot & $21.15(3.49)$ & $21.11(3.75)$ & $\mathrm{t}_{250}=0.08$ & $\mathrm{~ns}$ \\
\hline BIS-ImpNPlan & $27.47(4.61)$ & $28.02(4.34)$ & $\mathrm{t}_{260}=-0.98$ & $\mathrm{~ns}$ \\
\hline BIS-TOT & $65.95(8.80)$ & $65.79(8.13)$ & $\mathrm{t}_{233}=0.14$ & $\mathrm{~ns}$ \\
\hline YSR-ADHD & $56.31(8.03)$ & $54.07(6.91)$ & $\mathrm{t}_{267}=2.45$ & $<.05$ \\
\hline YSR-ODD & $57.39(6.46)$ & $56.29(6.34)$ & $\mathrm{t}_{267}=1.41$ & $\mathrm{~ns}$ \\
\hline YSR-CD & $58.21(9.07)$ & $54.44(6.75)$ & $\mathrm{t}_{267}=3.85$ & $<.05$ \\
\hline
\end{tabular}

Table 2: Means (standard deviations) for males and females together with the test statistics used for their comparison. *Given the nature of the variable e non parametric Wilcoxon test was conducted on the ranks. the BIS-11, indicating a greater impulsiveness $(\mathrm{F} 2,232=10.34 ; \mathrm{p}<05)$. This was also true when we considered the single subscales: attentional $(\mathrm{F} 2,254=3.42 ; \mathrm{p}<05)$, motor $(\mathrm{F} 2,259=6.42 ; \mathrm{p}<05)$ and non-planning $(\mathrm{F} 2,259=6.10 ; \mathrm{p}<05)$.

The same results emerged for the scores obtained in the YSR 1118: a higher alcohol consumption was significantly associated with an increase in oppositional-defiant disorder (F2,266 $=5.72 ; \mathrm{p}<.05)$ and conduct disorder $(\mathrm{F} 2,266=19.34 ; \mathrm{p}<.05)$, while there was no significant difference in relation to $\mathrm{ADHD}(\mathrm{F} 2,266=2.03 ; \mathrm{p}=$ n.s. $)$.

Using contrast analysis and dividing the whole sample into two groups, i.e. non-drinkers (group $0=0 \mathrm{AU}$ ) and drinkers (group $1=\geq 1$ $\mathrm{AU})$ [Contrast 1], there was a statistically significant difference for the impulsiveness construct [BIS TOT $(\mathrm{t} 232=-4.53 ; \mathrm{p}<.05)$ and subscales], for ADHD (t266=-1.99; $\mathrm{p}<.05)$, for ODD ( $\mathrm{t} 266=-3.34 ; \mathrm{p}<.05)$, and for $\mathrm{CD}(\mathrm{t} 266=-6.04 ; \mathrm{p}<05)$.

In a second contrast study, we divided the group of drinkers into group 1 (moderate drinkers=1-2 AU) and group 2 (heavy drinkers $\geq 3 \mathrm{AU}$ ). In this case, there was evidence of a statistically significant difference only inasmuch as concerned CD (t266=-4.12; $\mathrm{p}<05)$. Table 3 summarizes the results obtained for the two contrasts studies carried out on the whole sample.

In short, therefore: (i) adolescent drinkers were more impulsive than non-drinkers; and (ii) adolescent drinkers had higher scores for diagnoses of CD and ODD, while the findings relating to ADHD were statistically less noteworthy. Items (i) and (ii) were confirmed by contrast analysis, which emphasized statistically significant differences between groups 0 and 1 (young non-drinkers versus drinkers) for each subscale investigating the impulsiveness construct, i.e. attentional, motor, non-planning and total impulsiveness, CD, ODD, and ADHD. The same trend was expected to emerge from the contrast analysis between groups 1 and 2 (moderate versus heavy drinkers), but in this case there was only evidence of a statistical significance for CD.

Secondly, we investigated whether there were any significant differences between males and females in relation to their alcohol use and impulsiveness. For this purpose, we conducted descriptive analyses and ANOVA. To do so, we divided the sample into two groups by gender.

For males, the scores for impulsiveness [total $(F 2,117=6.6 ; \mathrm{p}<.05)$ and non-planning $(\mathrm{F} 2,124=6.7 ; \mathrm{p}<.05)]$ and for $\mathrm{CD}(\mathrm{F} 2,135=14.64$; $\mathrm{p}<.05)$ revealed significant differences depending on their reported alcohol consumption (0 AU, 1-2 AU, $\geq 3 \mathrm{AU})$.

For females too, there were significant differences on the scales for impulsiveness [total $(\mathrm{F} 2,112=3.78 ; \mathrm{p}<.05)$ and motor $(\mathrm{F} 2,122=5,99$; $\mathrm{p}<.05)]$, ODD (F2,128=3.67; $\mathrm{p}<.05)$, and $\mathrm{CD}(\mathrm{F} 2,128=3.67 ; \mathrm{p}<.05)$ correlating with their alcohol consumption.

\begin{tabular}{|c|c|c|c|c|c|c|c|c|}
\hline \multirow[b]{2}{*}{ Scale } & \multicolumn{4}{|c|}{ Contrast 1} & \multicolumn{4}{|c|}{ Contrast 2} \\
\hline & M g0 (sd) & M g1 (sd) & $\mathbf{t}$ & $p$ & M g1 (sd) & M g2 (sd) & $\mathbf{t}$ & $\mathbf{p}$ \\
\hline BIS-ImpAtt & $16.68(3.23)$ & $17.68(3.43)$ & -2.61 & $<.05$ & $17.51(3.19)$ & $18.32(4.43)$ & -1.08 & n.s. \\
\hline BIS-ImpMot & $20.42(3.44)$ & $21.98(3.61)$ & -3.51 & $<.05$ & 21.81 (3.69) & $22.56(3.53)$ & -0.94 & n.s. \\
\hline BIS-ImpNPlan & $26.91(4.35)$ & $28.70(4.43)$ & -3.47 & $<.05$ & $28.44(4.27)$ & $29.59(5.01)$ & -1.20 & n.s. \\
\hline BIS-TOT & $63.82(7.65)$ & $68.32(2.13)$ & -4.53 & $<.05$ & $67.61(8.16)$ & $70.79(10.42)$ & -1.68 & n.s. \\
\hline YSR-ADHD & $54.53(7.21)$ & $56.04(7.91)$ & -1.99 & $<.05$ & $55.63(8.27)$ & $57.58(6.44)$ & -1.17 & n.s. \\
\hline YSR-ODD & $55.72(5.86)$ & $58.20(6.91)$ & -3.34 & $<.05$ & $57.90(6.71)$ & $59.35(7.15)$ & -1.04 & n.s. \\
\hline YSR-CD & $54.36(7.35)$ & $58.77(8.51)$ & -6.04 & $<.05$ & $57.28(7.85)$ & $64.31(9.15)$ & -4.12 & $<.05$ \\
\hline
\end{tabular}

Table 3: Means (standard deviations) for whole sample and $t$ values for the contrast analysis. In Contrast 1 the two compared groups were the non drinkers (g0) versus drinkers (g1), while in Contrast 2 the moderate drinkers (g1) were compared to the heavy drinkers (g2). 
The contrast analyses were also conducted by gender.

Males, Contrast 1: when the sample was divided into two groups, non-drinkers (group $0=0 \mathrm{AU}$ ) versus drinkers (group $1=\geq 1 \mathrm{AU}$ ), there were statistically significant differences for the BIS TOT scale (t232=3.63; $\mathrm{p}<.05)$, for the subscale referring to non-planning impulsiveness $(\mathrm{t} 133=-3.64 ; \mathrm{p}<.05)$, and for $\mathrm{CD}(\mathrm{t} 135=-5.15 ; \mathrm{p}<.05)$.

Males, Contrast 2: when group 1 (moderate drinkers=1-2 AU) was compared with group 2 (heavy drinkers $\geq 3 \mathrm{AU}$ ), a statistically significant difference only emerged for $\mathrm{CD}(\mathrm{t} 135=-3.79 ; \mathrm{p}<.05)$.

Females, Contrast 1: in the comparison between groups 0 and 1 in this sub-sample, significant differences emerged for the BIS total scale $(\mathrm{t} 112=-2.77 ; \mathrm{p}<.05)$, and the motor impulsiveness subscale $(\mathrm{t} 122=-3.32$; $\mathrm{p}<.05)$, and for ODD (t128=-2.69; $\mathrm{p}<.05)$ and $\mathrm{CD}(\mathrm{t} 128=-2.57 ; \mathrm{p}<.05)$.

Females, Contrast 2: conducting the contrast analysis between group 1 (moderate drinkers $=1-2 \mathrm{AU}$ ) and group 2 (heavy drinkers $\geq 3$ $\mathrm{AU})$, a statistical significance only emerged for $\mathrm{CD}(\mathrm{t} 128=-1.96 ; \mathrm{p}=.05)$.

Table 4 summarizes the results of the two contrasts studies carried out separately for males and females.

\section{Discussion}

Recent research indicates that young people with impulsive tendencies are more likely to drink considerable amounts of alcohol in adolescent age and/or to have alcohol-related problems as they grow older [24]. This goes to show that although a greater impulsiveness was associated with alcohol consumption, drinking more heavily was not associated with any further increase in impulsiveness. It would therefore be wrong to claim that the adolescents who drank more heavily were more impulsive than those who drank more moderately. For conduct disorder, on the other hand, the inference emerging from the first contrast analysis still held. Our findings therefore suggest that impulsiveness and behavioral disorders are associated with alcohol consumption, with statistically significant differences between adolescents who drank and those who did not. In addition, we found a significant difference for conduct disorder between those who drank more or less heavily, higher scores for CD coinciding with higher levels of alcohol consumption.

Our findings show that impulsive traits are indeed associated with alcohol consumption, but higher levels of impulsiveness do not coincide with heavier drinking. This would seem to confirm the hypothesis that impulsive traits predispose individuals to alcohol use.

We also found evidence of gender-related differences in the impulsiveness subdomains involved, non-planned impulsiveness being more typical of males and motor impulsiveness of females. In fact the second part of our analysis conducted to ascertain the relationship between alcohol intake and impulsiveness and behavioral disorders by gender showed that this relationship was confirmed, albeit with some gender-related differences. For males the most significant findings associated with alcohol consumption related to impulsiveness (particularly non-planning) and CD; for females there were significant differences relating to impulsiveness (particularly motor) and to ODD, as well as CD. We thus concluded that, although the "gender" variable does not influence the global relationship between alcohol consumption and impulsiveness, it does seem to distinguish a different sensitivity to certain subdomains of impulsiveness and behavioral disorders. Male gender is more associated with the non-planning component of impulsiveness and with $\mathrm{CD}$, female gender with the motor component of impulsiveness and with ODD.

This gives the impression that, as far as alcohol use is concerned, a response inhibition deficit plays a major part in females' behavior, as opposed to a prevalent role of contextualization and planning difficulties in males. This data about gender-related impulsiveness components recalls studies on the brain's morphology and the neuropsychological effects of binge drinking in adolescent age which have demonstrated its association with specific gender-related differences in the cortical thickness of the frontal areas and in the quality of cognitive performance: females are more susceptible to the negative effects of alcohol on their neural development [25]. As for the psychopathological comorbidities, consistently with other studies $[7,9,25,26]$, our data show a significant difference in behavior in the sphere of conduct disorder between heavier and more moderate drinkers, higher alcohol consumptions coinciding with higher scores for CD. This picture could be seen as representing the behavioral correlates associated with impulsiveness and further exacerbated by the neurobiological effects of alcohol on the brain, and particularly on the regions that are still immature in preadolescence and adolescence, i.e. the sites of higher functions such as judgment, emotional control, self-control, organization and action planning $[27,28]$, functions that are typically affected in CD. Impulsiveness could thus be considered

\begin{tabular}{|c|c|c|c|c|c|c|c|c|c|}
\hline & \multirow[b]{2}{*}{ Scale } & \multicolumn{4}{|c|}{ Contrast 1} & \multicolumn{4}{|c|}{ Contrast 2} \\
\hline & & M g0 (sd) & M g1 (sd) & $t$ & p & M g1 (sd) & M g2 (sd) & $\mathbf{t}$ & p \\
\hline \multirow{7}{*}{ 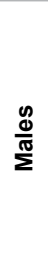 } & BIS-ImpAtt & $16.863 .52)$ & $17.86(3.54)$ & -1.92 & n.s. & $17.64(3.17)$ & $18.67(4.45)$ & -1.00 & n.s. \\
\hline & BIS-ImpMot & $20.63(3.64)$ & 21.60 (3.14) & -1.67 & n.s. & $21.47(3.46)$ & $22.07(2.87)$ & -0.58 & n.s. \\
\hline & BIS-ImpNPlan & $26.05(4.46)$ & $28.70(4.41)$ & -3.64 & $<.05$ & $28.39(4.36)$ & $29.81(4.58)$ & -1.14 & n.s. \\
\hline & BIS-TOT & $63.19(8.26)$ & $68.44(8.32)$ & -3.63 & $<.05$ & $67.73(8.72)$ & $70.93(7.90)$ & -1.25 & n.s. \\
\hline & YSR-ADHD & $55.06(9.34)$ & $57.42(5.84)$ & -1.72 & n.s. & $57.24(6.93)$ & $58.13(4.72)$ & -0.39 & n.s. \\
\hline & YSR-ODD & $56.35(6.47)$ & $58.31(6.40)$ & -1.85 & n.s. & $58.10(6.26)$ & $59.13(6.89)$ & -0.53 & n.s. \\
\hline & YSR-CD & $55.32(8.39)$ & 60.78 (8.23) & -5.15 & $<.05$ & $58.91(8.13)$ & $68.00(8.40)$ & -3.79 & $<.05$ \\
\hline \multirow{7}{*}{ 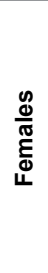 } & BIS-ImpAtt & 16.54 (2.99) & $17.42(3.84)$ & -1.51 & n.s. & $17.32(3.26)$ & $17.80(4.59)$ & -0.42 & n.s. \\
\hline & BIS-ImpMot & $20.27(3.31)$ & $22.51(4.12)$ & -3.32 & $<.05$ & $22.30(4.01)$ & $23.30(4.40)$ & -0.78 & n.s. \\
\hline & BIS-ImpNPlan & $27.60(4.15)$ & $29.29(5.04)$ & -1.45 & n.s. & 28.51 (4.99) & 29.27 (6.01) & -0.51 & n.s. \\
\hline & BIS-TOT & $64.32(7.14)$ & $68.15(10.27)$ & -2.77 & $<.05$ & $67.44(7.40)$ & $70.60(13.69)$ & -1.11 & n.s. \\
\hline & YSR-ADHD & $54.10(4.92)$ & $54.02(8.94)$ & -0.66 & n.s. & $53.23(9.53)$ & $56.82(8.45)$ & -1.52 & n.s. \\
\hline & YSR-ODD & $55.21(5.31)$ & 58.04 (7.59) & -2.69 & $<.05$ & $57.59(7.41)$ & $59.64(7.83)$ & -0.96 & n.s. \\
\hline & YSR-CD & $53.59(6.35)$ & $55.82(7.31)$ & -2.57 & $<.05$ & $54.85(6.81)$ & $59.27(7.89)$ & -1.96 & $=.05$ \\
\hline
\end{tabular}

Table 4: Means (standard deviations) for whole sample and $t$ values for the contrast analysis divided by gender. In Contrast 1 the two compared groups were the non drinkers (g0) versus drinkers ( $\mathrm{g} 1$ ), while in Contrast 2 the moderate drinkers ( $\mathrm{g} 1$ ) were compared to the heavy drinkers (g2). 
a factor that first predisposes adolescents to alcohol use and, due to the resulting neurobiological impairment in the frontal region, it would subsequently promote further behavioral issues attributable to CD and ODD. A useful model for explaining much of the behavior characteristic of CD refers to Koob's model [29,30] according to which drug addiction impacts multiple motivational mechanisms and can be conceptualized as a disorder that progresses from impulsivity (positive reinforcement) to compulsivity (negative reinforcement). The construct of negative reinforcement is defined as drug taking that alleviates a negative emotional state, which would change from the impulsive intake of a substance to seek gratification into a compulsive intake to avoid the negative somatic states deriving from a reduction of the pleasure $[29,30]$.

Our data about gender related behavior disorder (with CD prevailing in males and ODD in females) arouses some considerations. In fact while the literature supports a male prevalence of $\mathrm{CD}$, with or without any associated alcohol consumption [31-34] the prevailing tendency to develop ODD seen in our female adolescent sample is a novel finding. The reported rates of ODD range from $2 \%$ to $16 \%$, depending on the nature of the sample population and the methods used to assess the disorder, and generally with a higher prevalence among males, though the differences in the proportions identified between the two genders probably tend to disappear after puberty [35]. Our finding may consequently be of interest because it appears to link alcohol use and its effects in adolescence with particular psychopathologies, hinting at a possible alcohol-related tendency of the two genders to develop a given disorder. Recent studies on adolescents alcohol use disorder (AUD) have found that comorbidities that may interact with alcohol use and neural development, having different effects on males and females: males with AUD have a higher likelihood of showing signs of conduct disorder, while females with AUD smoke more cigarettes [36]. The authors suggest that such gender-related differences in the effects of alcohol on the brain derive from a different timing of the brain's prefrontal development in the two genders, a different gene expression that makes alcohol more neurotoxic in females, higher blood concentrations of alcohol in females than in males for the same amounts of alcohol ingested, and alcohol dehydrogenase levels [37].

\section{Limitations of the study and future developments}

First of all, an immediately obvious limitation of this study concerns the sample: though it was broad-based and sizeable, it still represents only a particular territorial area so our findings may not be generalizable (even for other parts of Italy).

Another limitation concerns the temporal domain because the study was based on data collected from questionnaires that were administered only once, without retesting the sample after a certain time interval (which might have enabled us to collect information on the trend of the relationship between impulsiveness and alcohol over time) [38].

From a methodological standpoint, moreover, it would have been interesting to conduct a case-control study and consider data derived from a clinical sample [39].

These issues will provide food for thought when it comes to further developing this area of research.

\section{Conclusions}

Despite some cited studies about neurobiology of alcohol abuse, researches concerning effects of alcohol misuse in youths in terms of behavior, particularly under the light of impulsivity, are lacking; and more, only a few papers try to study these issues paying attention to differentiate by levels of alcohol intake, as we have done

What emerges from this study is that there are marked and partly gender-related differences in terms of impulsiveness traits between adolescents who drink alcohol and those who do not. There are also differences in the psychopathologies potentially associated with heavy drinkers by comparison with those who drink more moderately, here again with some gender-related differences. The value of this study lies not only in its contribution, albeit with the above-mentioned limitations, towards our understanding of the mechanism (and hypothetical "vicious circle of reinforcement") lying behind the association between alcohol and behavioral disorders in adolescence, but also in its possible implications for the design of specific, differentiated prevention schemes for adolescents who have impulsive traits and would consequently be at higher risk of alcohol consumption and the related behavioral disorders. In this sense, impulsiveness could become the focus of measures in programs for the primary prevention of alcohol consumption (because it is a risk factor for adolescents' early alcohol consumption), while behavioral disorders and antisocial behavior could be the focus of secondary prevention programs on adolescent alcohol abuse. This approach would also be justified by the fact that, from the prognostic standpoint, there are reports of a rising prevalence and a greater severity and persistence of aggressive and destructive behavior, which is becoming manifest at an earlier age, and this consequently represents a major challenge in the field of mental health. From the developmental standpoint, the age of onset of antisocial behavior is a key issue because the extent of the influence of various causal factors varies with the age of onset of antisocial behavior and it has been suggested that a direct relationship exists between the time of onset and the severity of the resulting clinical picture [34].

\section{References}

1. Fossati A, Di Ceglie A, Acquarini E, Barratt ES (2001) Psychometric properties of an Italian version of the Barratt Impulsiveness Scale-11 (BIS-11) in nonclinical subjects. J Clin Psychol 57: 815-828.

2. Ivanova MY, Achenbach TM, Rescorla LA, Dumenci L, Almqvist F, et al. (2007) The generalizability of the Youth Self-Report syndrome structure in 23 societies. J Consult Clin Psychol 75: 729-738.

3. Spear LP (2010) the behavioral neuroscience of adolescence. W.W. Norton \& Company, New York, USA.

4. White AM, Swartzwelder HS (2005) Age-related effects of alcohol on memory and memory-related brain function in adolescents and adults. Recent Dev Alcohol 17: 161-176.

5. Nixon K, McClain JA (2010) Adolescence as a critical window for developing an alcohol use disorder: current findings in neuroscience. Curr Opin Psychiatry 23: $227-232$

6. Geier CF, Terwilliger R, Teslovich T, Velanova K, Luna B (2010) Immaturities in reward processing and its influence on inhibitory control in adolescence. Cereb Cortex 20: 1613-1629.

7. Gatta M, Cadevini C, Svanellini L, Aste G, Penzo M, Montagnese S, Schiff S, Battistella PA, Gatta A (2014) Psycho-behavioral vulnerability of adolescent binge drinkers. Prevention \& Research (in press).

8. Gallimberti L, Chindamo S, Buja A, Forza G, Tognazzo F., et al. (2011) Underage drinking on Saturday nights, sociodemographic and environmental risk factors: a cross-sectional study. Substance Abuse Treatment, Prevention, and Policy, 6: 15

9. Gatta, M, Dal Zotto, L, Schiff S, Svanellini, L., Lai J, Salis M, Penzo M, Gatto Rotondo C, Battistella PA, Gatta A (2012) Alcohol abuse and psychopathology in adolescence. Gionale di Neuropsichiatria dell'Età Evolutiva 32: 197-203.

10. Schweinsburg AD, McQueeny T Nagel BJ, Eyler LT, Tapert SF (2010) A preliminary study of functional magnetic resonance imaging response during verbal encoding among adolescent binge drinkers. Alcohol 44: 111-117.

11. McQueeny T, Schweinsburg BC, Schweinsburg AD, Jacobus J, Bava S, et al. 
Citation: Gatta M, Spoto A, Lamboglia S, Penzo M, Testa CP, et al. (2014) Impulsiveness, Behavioral Disorders and Alcohol Misuse in Teenage Students in Northern Italy. J Psychol Abnorm Child 3: 122. doi:10.4172/2329-9525.1000122

Page 6 of 6

(2009) Altered white matter integrity in adolescent binge drinkers. Alcohol Clin Exp Res 33: 1278-1285.

12. Maurage $P$, Joassin F, Speth A, Modave J, Philippot $P$, et al. (2012) Cerebral effects of binge drinking: respective influences of global alcohol intake and consumption pattern. Clin Neurophysiol 123: 892-901.

13. López-Caneda E, Cadaveira F, Crego A, Gómez-Suárez, A., Corral, M., et al. (2012) Hyperactivation of right inferior frontal cortex in young binge drinkers during response inhibition: a follow-up study. Addiction, 107, 1796-1808.

14. Barkataki I, Kumari V, Das M, Sumich A, Taylor P, et al. (2008) Neural correlates of deficient response inhibition in mentally disordered violent individuals. Behav Sci Law 26: 51-64.

15. Frick PJ, White SF (2008) Research review: the importance of callousunemotional traits for developmental models of aggressive and antisocial behavior. J Child Psychol Psychiatry 49: 359-375.

16. Jollant F, Guillaume S, Jaussent I, Bellivier F, Leboyer M, et al. (2007) Psychiatric diagnoses and personality traits associated with disadvantageous decision-making. Eur Psychiatry 22: 455-461.

17. Völlm B, Richardson P, McKie S, Elliott R, Dolan M, et al. (2007) Neuronal correlates of reward and loss in Cluster $B$ personality disorders: a functional magnetic resonance imaging study. Psychiatry Res 156: 151-167.

18. Yechiam E, Kanz JE, Bechara A, Stout JC, Busemeyer JR, et al. (2008) Neurocognitive deficits related to poor decision making in people behind bars. Psychon Bull Rev 15: 44-51.

19. Blair RJ (2009) Too much of a good thing: increased grey matter in boys with conduct problems and callous-unemotional traits. Brain 132: 831-832.

20. De Brito SA, Mechelli A, Wilke M, Laurens KR, Jones AP, et al. (2009) Size matters: increased grey matter in boys with conduct problems and callousunemotional traits. Brain 132: 843-852.

21. Huebner T, Vloet TD, Marx I, Konrad K, Fink GR, et al. (2008) Morphometric brain abnormalities in boys with conduct disorder. J Am Acad Child Adolesc Psychiatry 47: 540-547.

22. Patton JH, Stanford MS, Barratt ES (1995) Factor structure of the Barratt Impulsiveness Scale. Journal of Clinical Psychology 51: 768-74.

23. Frigerio A, Vanzin L, Pastore V, Nobile M, Giorda R, et al. (2006) The Italian preadolescent mental health project (PrISMA): rationale and methods. Int $J$ Methods Psychiatr Res 15: 22-35

24. Fernie G, Peeters M, Gullo MJ, Christiansen P, Cole JC, et al. (2013) Multiple behavioural impulsivity tasks predict prospective alcohol involvement in adolescents. Addiction 108: 1916-1923.

25. Squeglia LM, Schweinsburg AD, Pulido C, Tapert SF (2011) Adolescent binge drinking linked to abnormal spatial working memory brain activation: differential gender effects. Alcoholism: Clinical and Experimental Researc, 35: 1-11.

26. Townshend JM, Kambouropoulos N, Griffin A, Hunt FJ, Milani RM (2014) Binge drinking, reflection impulsivity, and unplanned sexual behavior: impaired decision-making in young social drinkers. Alcohol Clin Exp Res 38: 1143-1150.

27. Gardner M, Steinberg L (2005) Peer influence on risk taking, risk preference, and risky decision making in adolescence and adulthood: an experimental study. Dev Psychol 41: 625-635.

28. Volkow ND, Li TK (2004) Drug addiction: the neurobiology of behaviour gone awry. Nat Rev Neurosci 5: 963-970.

29. Koob GF (2009) Neurobiological substrates for the dark side of compulsivity in addiction. Neuropharmacology 56 Suppl 1: 18-31.

30. Koob GF (2013) Addiction is a Reward Deficit and Stress Surfeit Disorder. Front Psychiatry 4: 72 .

31. Kazdin AE (1995) Conduct disorders in childhood and adolescence. Developmental Clinical Psychology and Psychiatry, Vol.9. Sage Publications London.

32. Cohen S, Tyrrell DA, Smith AP (1993) Negative life events, perceived stress, negative affect, and susceptibility to the common cold. J Pers Soc Psychol 64: $131-140$.

33. Russo MF, Beidel DC (1994) Comorbidity of childhood anxiety and externalizing disorders: Prevalence, associated characteristics, and validation issues. Clinical Psychology Review, 14: 199-221.

34. Farruggia R, Romani M, Bartolomeo S (2008). Disturbi della Condotta/Disturb della Personalità: riflessioni teorico-cliniche per una presa in carico precoce. Psichiatria dell'Infanzia e dell'Adolescenza, 75: 503-514.

35. Medina KL, McQueeny T, Nagel BJ, Hanson KL, Schweinsburg AD, et al. (2008) Prefrontal cortex volumes in adolescents with alcohol use disorders: unique gender effects. Alcohol Clin Exp Res 32: 386-394.

36. Roskam I, Stievenart M, Tessier R, Muntean A, Escobar MJ, et al. (2014) Another way of thinking about ADHD: the predictive role of early attachment deprivation in adolescents' level of symptoms. Soc Psychiatry Psychiatr Epidemiol 49: 133-144.

37. Cohen S, Tyrrell DA, Smith AP (1993) Negative life events, perceived stress negative affect, and susceptibility to the common cold. J Pers Soc Psychol 64 131-140.

38. De Brito SA, Mechelli A, Wilke M, Laurens KR, Jones AP, et al. (2009) Size matters: increased grey matter in boys with conduct problems and callousunemotional traits. Brain 132: 843-852.

39. Galvan A (2010) Adolescent development of the reward system. Front Hum Neurosci 4: 6.
Citation: Gatta M, Spoto A, Lamboglia S, Penzo M, Testa CP, et al. (2014) Impulsiveness, Behavioral Disorders and Alcohol Misuse in Teenage Students in Northern Italy. J Psychol Abnorm Child 3: 122. doi:10.4172/2329 9525.1000122

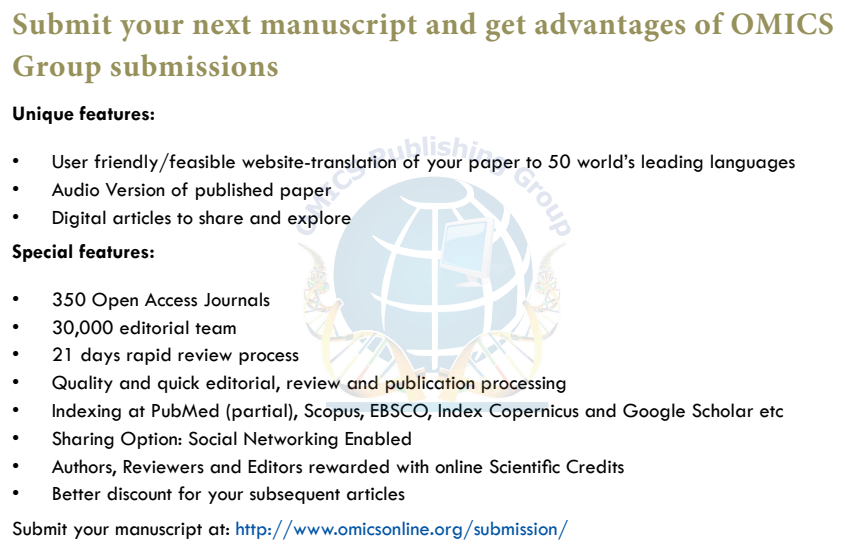

